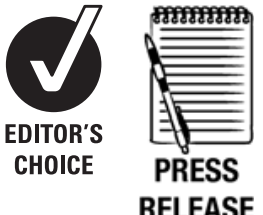

RELEASE

\section{See Editorial, p 1787}

${ }^{1}$ Department of Cardiology, Royal Hallamshire Hospital, Sheffield, UK

${ }^{2}$ Academic Unit of Diabetes Endocrinology and Metabolism University of Sheffield Medical School, Sheffield, UK ${ }^{3}$ Centre for Diabetes and Endocrinology, Barnsley Hospital, Barnsley, UK

\section{Correspondence to} Professor Kevin S Channer, Department of Cardiology, M131, Royal Hallamshire Hospital, Glossop Road, Sheffield S10 2JF, UK; kevin.channer@sth.nhs.uk

Accepted 3 June 2010

\title{
Low serum testosterone and increased mortality in men with coronary heart disease
}

\author{
Chris J Malkin, ${ }^{1}$ Peter J Pugh, ${ }^{1}$ Paul D Morris, ${ }^{1}$ Sonia Asif, ${ }^{1}$ T Hugh Jones, ${ }^{2,3}$ \\ Kevin S Channer ${ }^{1}$
}

\begin{abstract}
Background To examine the effect of serum testosterone levels on survival in a consecutive series of men with confirmed coronary disease and calculate the prevalence of testosterone deficiency.

Design Longitudinal follow-up study.

Setting Tertiary referral cardiothoracic centre.

Patients 930 consecutive men with coronary disease referred for diagnostic angiography recruited between June 2000 and June 2002 and followed up for a mean of $6.9 \pm 2.1$ years
\end{abstract}

Outcome All-cause mortality and vascular mortality. Prevalence of testosterone deficiency.

Results The overall prevalence of biochemical testosterone deficiency in the coronary disease cohort using bio-available testosterone (bio-T) $<2.6 \mathrm{nmol} / \mathrm{l}$ was $20.9 \%$, using total testosterone $<8.1 \mathrm{nmol} / \mathrm{l}$ was $16.9 \%$ and using either was $24 \%$. Excess mortality was noted in the androgen-deficient group compared with normal (41 (21\%) vs 88 (12\%), $p=0.002$ ). The only parameters found to influence time to all-cause and vascular mortality ( $\mathrm{HR} \pm 95 \% \mathrm{Cl})$ in multivariate analyses were the presence of left ventricular dysfunction $(3.85 ; 1.72$ to 8.33), aspirin therapy (0.63; 0.38 to 1.0$), \beta$-blocker therapy $(0.45 ; 0.31$ to 0.67$)$ and low serum bio-T (2.27; 1.45 to 3.6$)$.

Conclusions In patients with coronary disease testosterone deficiency is common and impacts significantly negatively on survival. Prospective trials of testosterone replacement are needed to assess the effect of treatment on survival.

Men have more than twice the risk of dying from coronary disease than women, an effect that persists after controlling for cardiovascular risk factors. ${ }^{1}$ It has been assumed that testosterone is deleterious to the male cardiovascular system and contributes to the vascular risk. In fact, there is little evidence that endogenous testosterone is an adverse risk factor but the role of testosterone status and replacement therapy on male health is controversial. ${ }^{2}$

High doses of exogenous anabolic steroids are undoubtedly associated with cardiac disease, ${ }^{3}$ but high endogenous testosterone levels within the normal range do not appear to be harmful. Indeed, low rather than high testosterone levels in men are associated with several cardiovascular risk factors including an atherogenic lipid profile, insulin resistance, obesity and a prothrombotic fibrinolytic profile. ${ }^{4} 5$ Studies in male animals have shown that castration or induced hypogonadism increases atherosclerosis and testosterone replacement abrogates this. ${ }^{6}$ In addition, testosterone has beneficial effects in men with cardiac disease.
Testosterone is a potent coronary vasodilator, an effect mediated by a calcium channel antagonist action, and consequently has benefits on the angina threshold, particularly in men with low baseline testosterone. ${ }^{8} 9$ Testosterone therapy reduces total cholesterol, fat mass, waist circumference and pro-inflammatory cytokines associated with atherosclerosis, diabetes and the metabolic syndrome. ${ }^{10-12}$ Testosterone also improves functional capacity and insulin resistance in men with heart failure. ${ }^{13} 14$

In an ageing male population low serum total testosterone is common and has a prevalence of $30 \%$ in men over the age of 60 years. ${ }^{15}$ Testosterone deficiency may cause undesirable effects such as loss of bone and lean body mass, increased adiposity, low energy and impaired physical and sexual function. Until recently, these effects were viewed as the natural physiology of ageing; however, four recent major studies have found low testosterone to be associated with increased allcause mortality after controlling for baseline morbidity and age. ${ }^{16-19}$ The effect of testosterone on mortality is independent after adjustment for baseline covariates; the EPIC-Norfolk study ${ }^{17}$ excluded men with cardiovascular disease and cancer at baseline and yet found that all-cause mortality and cardiovascular mortality was increased for every $6 \mathrm{nmol} / \mathrm{l}$ reduction in serum testosterone. Low testosterone status is therefore associated with mortality and vascular mortality, yet no study has specifically examined patients with established cardiovascular disease. This is important because men with manifest coronary artery disease are at a higher risk of cardiovascular mortality and represent a patient population prone to testosterone deficiency. In addition, those men with angina, chronic heart failure or diabetes may derive particular symptomatic benefit from androgen replacement therapy. ${ }^{10} 122021$

This study had two aims, first to assess the impact of testosterone status on all-cause mortality in men with pre-existing coronary disease, and second to identify the prevalence of biochemical testosterone deficiency in men with coronary disease. Our hypothesis was that low serum testosterone would be associated with an adverse survival.

\section{METHODS \\ Subjects and design}

This was a prospective follow-up cohort study; subjects were recruited from male patients undergoing elective coronary angiography in a tertiary cardiac referral centre between June 2000 and June 2002. The study was approved by the local research 
ethics committee and all subjects gave written informed consent. Patients were approached before 09:30 hours on the morning of the procedure and asked to complete a questionnaire detailing history and medication. Height and weight were measured and body mass index (BMI) calculated, venous blood was taken for the same day measurement of C-reactive protein (CRP) and the remaining serum was sealed and frozen at $-20^{\circ} \mathrm{C}$ until assayed.

Patients were excluded from the study if they had malignancy, chronic inflammatory disease, myocardial infarction within the past 3 months, recent or current infection or uncontrolled heart failure, as these conditions are all associated with the suppression of testosterone production, or if they were taking sex hormone manipulating therapy. Similarly, in a predefined protocol exclusion, subjects were also excluded from further analysis if they had a serum CRP level above $10.0 \mathrm{mg} / \mathrm{l}$, even in the absence of clinical evidence of inflammation, because inflammatory disorders are associated with the suppression of testosterone production.

The result of each angiogram was reported by the patient's cardiologist at the time of the angiogram and before any measurement of the testosterone level. Significant coronary artery disease was defined as $70 \%$ or greater stenosis in any epicardial coronary artery or $50 \%$ or greater stenosis of the main stem of the left coronary artery. Coronary plaque was defined as diffuse coronary atheroma not fulfilling these criteria. Normal coronary arteries were defined as smooth coronary vessels free of surface irregularities or stenoses. Left ventricular function was classified as normal or as mild, moderate or severely impaired by assessment of left ventriculography at the time of angiography or by echocardiography using standard echocardiographic techniques. Patients with normal coronary arteries were excluded from the main analyses. Serum levels of total testosterone (TT) and bio-available (bio-T) testosterone were determined. Bioavailable testosterone was used because this value measures the free and albumen-bound testosterone and is a more accurate assessment of the biologically active component than total testosterone, especially in older men. ${ }^{22}$

It is recognised that anticipatory stress may affect testosterone levels. To determine the validity and reproducibility of hormone testing on the day of angiography, the first 50 eligible individuals were asked to return to the hospital outpatient department between 08:00 and 09:30 hours, 2 weeks after angiography. Blood was taken for repeat measurement of TT, and comparison made with the sample taken on the day of angiography. In addition, subject anxiety was assessed by the widely used Spielberger state-trait anxiety inventory, form $Y$ and the cognitive-somatic anxiety questionnaire. These assess individual stable anxiety-proneness. The difference between the testosterone level on the day of angiography and at follow-up was correlated with anxiety scores to assess whether patients' tendency to perceived stress was associated with falsely low testosterone on the day of angiography.

Patient data were electronically flagged for death certification at the Office of National Statistics, UK. All death certificates issued in the UK on study patients were copied to the research office. The information available was limited to patient details, date of death, postmortem reports (if performed) and the given cause of death by the certifying physician. In October 2008 after 145 deaths, the database was subject to the present analysis. Vascular mortality was defined as any death in which the primary cause was related to atherosclerotic vascular disease, heart failure or unheralded cardiac arrest (International Classification of Disease (version 10) codes I20-25, I70-71, I42.0, I46 and I50).
Hypogonadism is a clinical syndrome complex comprising symptoms and biochemical evidence of testosterone deficiency. ${ }^{23}$ Laboratory ranges differ between hospitals but at our institution, testosterone replacement is recommended in men with a total TT level less than $8.1 \mathrm{nmol} / 1$ or a bio-T testosterone level less than $2.6 \mathrm{nmol} / 1$. Therefore hypogonadism was defined as a bio-T level of less than $2.6 \mathrm{nmol} / 1$ or a TT level of less than $8.1 \mathrm{nmol} / \mathrm{l}$. The range of TT less than $15.1 \mathrm{nmol} / \mathrm{l}$ was also analysed as this group with borderline low total testosterone levels require further evaluation in standard endocrine practice.

\section{Assays}

Serum TT was measured in duplicate by ELISA (DRG Instruments Gmbh, Germany). Bio-T was measured by the method of Tremblay and Dube. ${ }^{24}$ CRP was measured by routine hospital assay. The intra-assay and interassay coefficients of variation of the assays were: TT less than $6 \%$ and less than $5 \%$; bio-T less than $5 \%$ and less than $8 \%$.

\section{Statistical analysis}

The relationship between testosterone status and mortality was determined using Cox proportional hazard regression. Initial unadjusted analyses were performed; each covariate was then added to the model to examine the effect on mortality. Finally, a multivariate regression model was constructed using all variables in a forward stepwise approach to determine which factors had influence after adjustment. Survival curves adjusted for these baseline variables were constructed to illustrate the effect of testosterone on mortality. The prevalence of hypogonadism was calculated from the raw data. Clinical predictors of biochemical hypogonadism were determined by regression. Categorical data were compared between groups using the $\chi^{2}$ test. Values are expressed as a percentage of each group or as mean \pm SD unless stated. Correlations were performed using Spearman's rank correlation ( $\mathrm{rS}$ ) or Pearson correlation ( $\mathrm{rP}$ ) as appropriate. All analyses were performed using SPSS version 14.5. Significance was sought at the 0.05 level.

\section{RESULTS}

\section{Reproducibility/reliability of the index blood test}

In the first 50 subjects with coronary artery disease, the TT level was $12.4 \pm 6.2 \mathrm{nmol} / \mathrm{l}$. On repeat testing, the mean testosterone level was $12.2 \pm 5.0 \mathrm{nmol} / \mathrm{l}, \mathrm{p}=0.72$ with close correlation between the two samples $(\mathrm{rP}=0.89, \mathrm{p}=0.0002)$. There was a strong correlation between the Spielberger state-trait anxiety inventory and cognitive-somatic anxiety questionnaire scores $(r S=0.70, p<0.01)$ but no relation of either score with the difference between the testosterone level on the day of angiography and at repeat testing $(\mathrm{r} S=-0.20, \mathrm{p}=0.30 ; \mathrm{rS}=-0.30, \mathrm{p}=0.20)$. The validity of hormone testing on the day of angiography was therefore accepted.

\section{Baseline data}

A total of 1165 eligible men was approached, 17 refused to participate so 1148 men were recruited, 70 were excluded from all analyses because of elevated CRP ( $>10 \mathrm{nmol} / \mathrm{l})$ and 148 were excluded from the primary predetermined analyses because of normal coronary arteries. This left a cohort of 930 men on whom the main analyses were performed. These men were followed up for a mean of $6.9 \pm 2.1$ years after recruitment. In the coronary disease group there were 129 deaths, of which 73 were vascular deaths; the equivalent figures for the non-coronary disease group were 16 and eight. The baseline data are shown in table 1 , this is further divided into men with bio-T less than $2.6 \mathrm{nmol} / \mathrm{l}$-a level specific for testosterone deficiency. 
Table 1 Baseline data

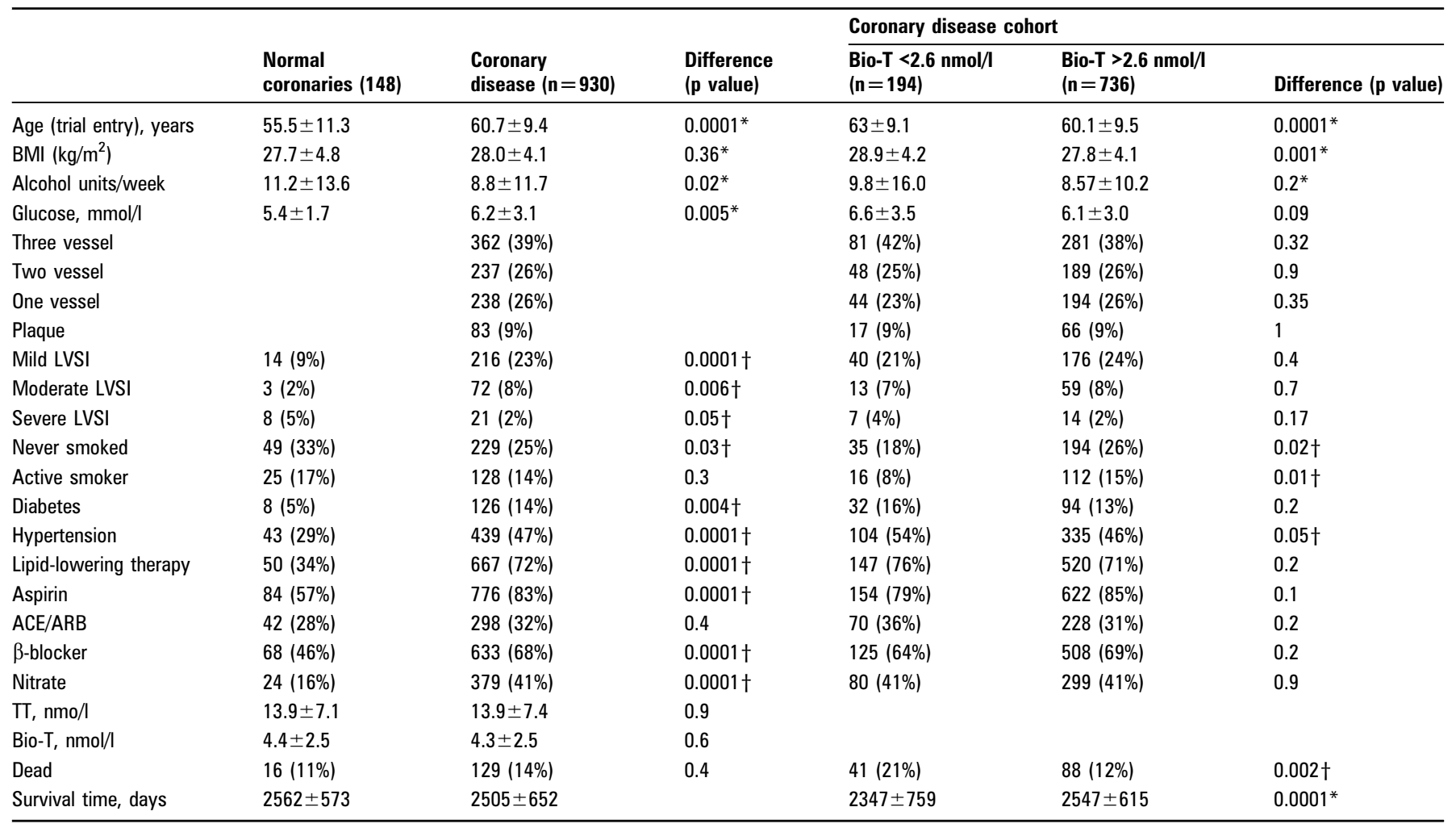

\footnotetext{
${ }^{*}$ Analysis by $\mathrm{t}$ test.

†By $\chi^{2}$.

ACE, angiotensin-converting enzyme; ARB, angiotensin II receptor blocker; BMI, body mass index; bio-T, bio-available testosterone; LVSI, left ventricular systolic impairment; TT, total
} testosterone.

\section{Impact on mortality}

In the multivariate analysis using all baseline variables in a forward conditional regression model the only parameters found to influence time to all-cause mortality ( $\mathrm{HR} \pm 95 \% \mathrm{CI}$ ) were left ventricular dysfunction ( $3.85 ; 1.72$ to 8.33$)$, aspirin therapy $(0.63 ; 0.38$ to 1.0$), \beta$-blocker therapy $(0.45 ; 0.31$ to 0.67$)$ and low serum bio-T (2.27; 1.45 to 3.6$)$. An adjusted survival curve of patients with low baseline bio-T $(<2.6 \mathrm{nmol} / \mathrm{l})$ shows a significant hazard imparted by a low bio-T level for all-cause (figure 1) and vascular mortality (figure 2). The full analysis was repeated on the whole cohort including those men with normal coronary arteries. The results were substantially the same except the presence of normal coronaries was associated with a favourable $\operatorname{HR}(0.56 ; 0.31$ to $1, p=0.05)$.

\section{Total testosterone and mortality}

In the multivariate analysis the serum total testosterone was inversely associated with mortality TT $(0.96 ; 0.9$ to 1$)$. A baseline TT level of less than $15.1 \mathrm{nmol} / 1$ showed an increased allcause hazard of 1.86 (1.1 to 3.2) and vascular mortality of 2.5 (1.2 to 5.3). However, the conventional cut-off of TT of less than $8.1 \mathrm{nmol} / 1$ did not demonstrate a significant survival hazard in the coronary disease cohort for all-cause mortality although this value approached significance 1.6 (0.95 to 2.85 ).

\section{Prevalence of hypogonadism in men with coronary disease}

The overall prevalence of biochemical testosterone deficiency in the coronary disease cohort using bio-T less than $2.6 \mathrm{nmol} / \mathrm{l}$ was $20.9 \%$, using TT less than $8.1 \mathrm{nmol} / \mathrm{l}$ was $16.9 \%$ and using either was $24 \%$.

On logistic regression with all variables included, predictors of low serum bio-T $(<2.6 \mathrm{nmol} / \mathrm{l})$ were age (OR 1.03; 1.0 to 1.1, $\mathrm{p}=0.003$ ), BMI (OR 1.06; 1.0 to $1.1, \mathrm{p}=0.004$ ) and former smoking (OR $0.59 ; 0.4$ to $0.9, \mathrm{p}=0.005$ ). The predictor of a low TT was only BMI (OR 1.14; 1.1 to $1.2, \mathrm{p}=0.0001)$. Predictors of

Survival by Testosterone Status. Bio-T I all cause mortality. [adjusted]

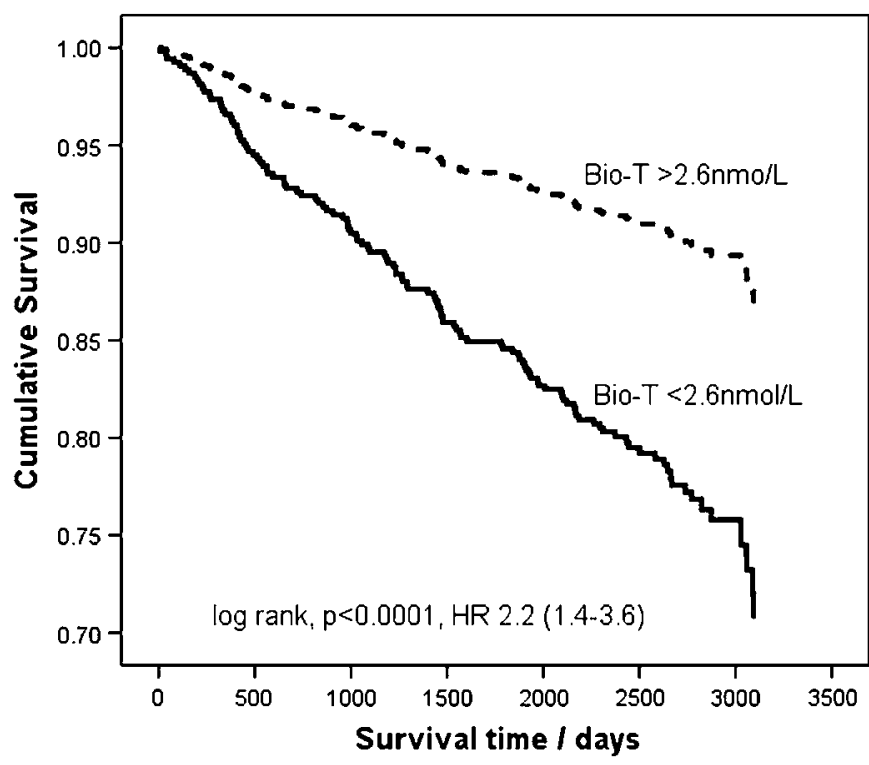

Figure 1 Shows a survival curve of all-cause mortality based on baseline bio-available testosterone (bio-T). The solid line represents patients with baseline bio-T less than $2.6 \mathrm{nmol} / \mathrm{l}$, the broken line represents patients with bio-T greater than $2.6 \mathrm{nmol} / \mathrm{l}$. HR, hazard ratio. 
Survival by Testosterone Status. Bio-T I vascular mortality. [adjusted]

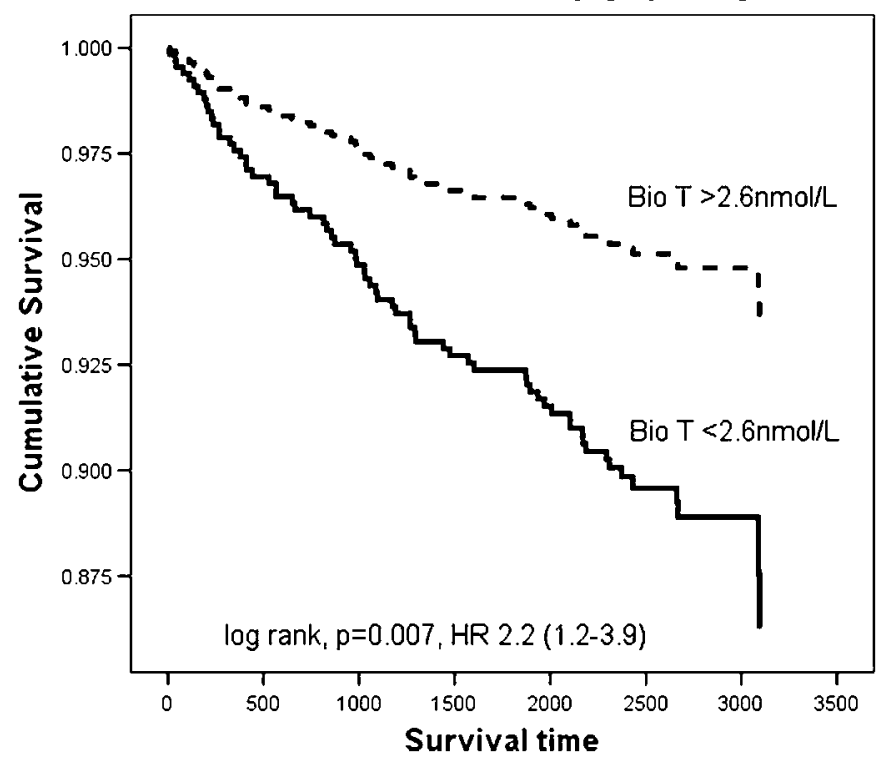

Figure 2 Shows a survival curve of vascular mortality based on baseline bio-available testosterone (bio-T). The solid line represents patients with baseline bio-T less than $2.6 \mathrm{nmol} / \mathrm{l}$, the broken line represents patients with bio-T greater than $2.6 \mathrm{nmol} / \mathrm{l}$. HR, hazard ratio.

either low TT or bio-T were age (OR 1.02; 1.0 to $1.04, \mathrm{p}=0.04$ ), BMI (OR 1.09; 1.04 to $1.13, \mathrm{p}=0.0001)$ and former smoking (OR $0.64 ; 0.5$ to $0.9, \mathrm{p}=0.01$ ).

Comparison of the coronary with the non-coronary disease groups found no significant difference in the prevalence of biochemical testosterone deficiency; ( $22 \%$ vs $20.9 \%$ for bio-T $<2.51 \mathrm{nmol} / \mathrm{l}, 19 \%$ vs $14.1 \%$ for TT $<8.1 \mathrm{nmol} / \mathrm{l}, 28 \%$ vs $24 \%$ for either; $\chi^{2}$ in each case $p>0.13$ ).

\section{DISCUSSION}

Our data suggest that the prevalence of biochemical testosterone deficiency is common in men with coronary disease and is present in $24 \%$ of this sample. The present data have confirmed that low endogenous bio- $\mathrm{T}$ is related to all-cause and vascular mortality in a coronary disease population. TT is weakly associated with survival and a cut-off of TT of less than $15.1 \mathrm{nmol} / 1$ is related to increased mortality. Therefore, we also conclude that borderline low levels of TT may also adversely impact on survival. The relationship of low bio-T to mortality is significant with only $\beta$-blocker therapy and left ventricular dysfunction having greater statistical influence on survival in the multivariate model. Importantly, the impact on survival was maintained after controlling for all baseline factors such as age, BMI, coexistent medication comorbidity and smoking. Testosterone levels themselves were significantly associated with BMI, age and former cigarette smoking, but the effect on mortality was still present after adjustment for these factors.

What is the implication of this finding? Our data cannot determine if low testosterone levels cause increased mortality or are merely a marker of risk due to underlying disease. Testicular function is suppressed in many acute and chronic diseases; our study did not have a healthy control group because the normal coronary artery group had a high level of valvular disease, left ventricular dysfunction and other comorbidities such as hypertension and diabetes. However, at inclusion, few if any subjects were acutely ill as they were selected before day case elective angiography; subjects with recent acute coronary syndromes, uncontrolled heart failure or elevated CRP were excluded. Within the confines of our data the severity of underlying disease does not influence endogenous serum testosterone as there was no effect on testosterone levels imparted by increasing the number of diseased coronary vessels or the presence and severity of left ventricular function. We have not measured the total burden of coronary atheroma but this is reported to be related to testosterone levels in one study. ${ }^{25}$

Our data expand on and are entirely consistent with previous studies of testosterone status on mortality. In previous studies the exclusion of patients with cardiovascular disease or the exclusion of all deaths within the first $5^{19}$ or 2 years ${ }^{17}$ did not weaken the relationship of low testosterone with mortality, in fact the HR increased. The inference from these data is that testosterone is not just a marker of poor health-should this be the case the relationship of testosterone and mortality ought to weaken following the exclusion of early deaths, whereas the reverse is seen. Our data confirm that high-risk patients with documented vascular disease are subject to similar hazards associated with low serum testosterone in other studies.

What is the pathophysiology of low testosterone status and the apparent increased mortality of atherosclerotic disease? Animal data show that testosterone deficiency accelerates atheroma and replacement prevents this; ${ }^{6} 7$ human follow-up studies have shown an increased progression of atheroma in men with lower testosterone. ${ }^{26-28}$ These data therefore suggest that testosterone deficiency is associated with progressive atherosclerosis and replacement, in animals at least, prevents this; the precise mechanism by which testosterone impacts on atheroma remains unclear.

Abnormal testosterone status is common, the prevalence of biochemical testosterone deficiency in the coronary disease cohort was $20.9 \%$ using bio-T less than $2.6 \mathrm{nmol} / 1,16.91 \%$ using TT less than $8.1 \mathrm{nmol} / 1$ and $24 \%$ using either, a figure similar to other studies. ${ }^{15}$ The present data also raise the issue of borderline testosterone deficiency. We have shown an excess mortality in men with TT of less than $15.1 \mathrm{nmol} / \mathrm{l}$, a level higher than most accepted definitions of hypogonadism. It is recognised that TT is not a specific test to determine androgen status because the total testosterone assay includes free testosterone, the loosely bound albumen fraction and the relatively tightly bound (inactive) sex hormone-binding globulin fraction. Bio-T seems to be more specific for detecting pathological deficiency and mortality as low levels less than $2.6 \mathrm{nmol} / 1$ predict mortality, whereas borderline levels do not. A low level of bio-T $(<2.6 \mathrm{nmol} / \mathrm{l})$ is therefore likely to be abnormal, whereas a high level of TT $(>15.1 \mathrm{nmol} / \mathrm{l})$ is likely to be normal. An indeterminate TT $(8.1-15.1 \mathrm{nmol} / \mathrm{l})$ is difficult to interpret: the bio-available testosterone may be pathologically low or normal. In line with accepted practice, we advocate assays to measure free or bioavailable testosterone in this subset. If these are not available formulae have been derived to estimate these, but these should be validated with the local laboratory. ${ }^{9} 29$

We have demonstrated that testosterone deficiency is associated with premature death in a cohort of patients with vascular disease; many of these patients died and will continue to die from cardiovascular disease. There is ongoing debate over the risk-benefit ratio of testosterone replacement in patients who are testosterone deficient, and there is still a concern among doctors that testosterone replacement therapy might increase the risk of vascular disease. Despite this there has been a huge increase in testosterone prescriptions in the past two decades, ${ }^{30}$ and there is scientific plausibility and a plethora of trials 
showing benefit in terms of risk factor modification and symptoms (reviewed in Jones et al). ${ }^{4}$ If androgen deficiency is part of the underlying pathophysiology of atherosclerotic disease in men, then the serum testosterone level could be viewed as a modifiable risk factor. Physiological testosterone replacement is an inexpensive and well-tolerated therapy but does require careful monitoring. ${ }^{23}$

A prospective outcome study of testosterone replacement on mortality is the logical next step. These trials should target highrisk populations such as men with cardiac disease or diabetes because these men have most to gain, their event rates are higher and any influence of therapy positive or adverse is more likely to be demonstrated within the confines of a clinical trial.

\section{Limitations}

This was a longitudinal follow-up study; the data from our subjects were only recorded at the time of angiogram and at death. We do not have data on subsequent revascularisations performed, changes in pharmacotherapy, insulin resistance, mood or vascular events other than related to the index mortality. It is possible that any or all of these may influence both mortality and serum androgen levels. In addition, patients may have been commenced on testosterone replacement therapy by other physicians although this number is likely to be very small.

Acknowledgements The authors thank the following colleagues for permitting the recruitment of their patients into the study: Dr W Baig, Dr RJ Bowes, Dr S Campbell, Dr J Gunn, Dr R Muthusamy, Dr C Newman, Dr GDG Oakley, Dr L O'Toole, Dr G Payne, Dr W Rhoden, Dr BT Saeed, Dr JN West and Dr NM Wheeldon. The authors also wish to acknowledge Miss Joanne Nettleship and Miss Joanne Hall for performing the majority of the total and bio-available testosterone assays.

Funding This study was funded by the South Sheffield Charitable Trust.

Competing interests None.

Patient consent Obtained.

Ethics approval This study was conducted with the approval of the South Yorkshire local research and ethics committee.

Contributors CJM collected the mortality data, analysed the final results and wrote the first draft of the paper. PJP designed the initial study, processed the ethics approval and collected baseline data. PDM helped design the initial study and collected the baseline data. SA helped design the initial study and collected the baseline data. THJ and KSC developed the original concept for the study, obtained funding and supervised ethics submission, data collection and writing of the final paper.

Provenance and peer review Not commissioned; externally peer reviewed.

\section{REFERENCES}

1. Njolstad I, Arnesen E, Lund-Larsen PG. Smoking, serum lipids, blood pressure, and sex differences in myocardial infarction. A 12-year follow-up of the Finnmark Study. Circulation 1996:93:450-6.

2. Haddad RM, Kennedy CC, Caples SM, et al. Testosterone and cardiovascular risk in men: a systematic review and meta-analysis of randomized placebo-controlled trials. Mayo Clin Proc 2007;82:29-39.

3. Bagatell CJ, Bremner WJ. Androgens in men - uses and abuses. N Eng/ J Med 1996;334:707-14.

4. Jones RD, Nettleship JE, Kapoor D, et al. Testosterone and atherosclerosis in aging men: purported association and clinical implications. Am J Cardiovasc Drugs 2005:5:141-54.
5. Kapoor D, Aldred H, Clark S, et al. Clinical and biochemical assessment of hypogonadism in men with type 2 diabetes: correlations with bioavailable testosterone and visceral adiposity. Diabetes Care 2007;30:911-17.

6. Alexandersen $\mathbf{P}$, Haarbo J, Byrjalsen I, et al. Natural androgens inhibit male atherosclerosis: a study in castrated, cholesterol-fed rabbits. Circ Res 1999:84:813-19.

7. Nettleship JE, Jones TH, Channer KS, et al. Physiological testosterone replacement therapy attenuates fatty streak formation and improves high-density lipoprotein cholesterol in the Tfm mouse: an effect that is independent of the classic androgen receptor. Circulation 2007;116:2427-34

8. Scragg JL, Jones RD, Channer KS, et al. Testosterone is a potent inhibitor of L-type $\mathrm{Ca}(2+)$ channels. Biochem Biophys Res Commun 2004;318:503-6.

9. Malkin CJ, Pugh PJ, Morris PD, et al. Testosterone replacement in hypogonadal men with angina improves ischaemic threshold and quality of life. Heart 2004:90:871-6.

10. Kapoor D, Goodwin E, Channer KS, et al. Testosterone replacement therapy improves insulin resistance, glycaemic control, visceral adiposity and hypercholesterolaemia in hypogonadal men with type 2 diabetes. Eur $\mathrm{J}$ Endocrinol 2006;154:899-906

11. Malkin CJ, Pugh PJ, Jones RD, et al. The effect of testosterone replacement on endogenous inflammatory cytokines and lipid profiles in hypogonadal men. J Clin Endocrinol Metab 2004;89:3313-18.

12. Heufelder $\mathbf{A E}$, Saad F, Bunck MC, et al. 52-Week treatment with diet and exercise plus transdermal testosterone reverses the metabolic syndrome and improves glycaemic control in men with newly diagnosed type 2 diabetes and subnormal plasma testosterone. J Androl 2009;30:726-33.

13. Malkin CJ, Jones RD, Jones TH, et al. Effect of testosterone on ex vivo vascular reactivity in man. Clin Sci (Lond) 2006:111:265-74.

14. Malkin CJ, Jones TH, Channer KS. The effect of testosterone on insulin sensitivity in men with heart failure. Eur J Heart Fail 2007;9:44-50.

15. Harman SM, Metter EJ, Tobin JD, et al. Longitudinal effects of aging on serum total and free testosterone levels in healthy men. Baltimore Longitudinal Study of Aging $J$ Clin Endocrinol Metab 2001:86:724-31.

16. Shores MM, Moceri VM, Gruenewald DA, et al. Low testosterone is associated with decreased function and increased mortality risk: a preliminary study of men in a geriatric rehabilitation unit. J Am Geriatr Soc 2004;52:2077-81.

17. Khaw KT, Dowsett M, Folkerd E, et al. Endogenous testosterone and mortality due to all causes, cardiovascular disease, and cancer in men: European prospective investigation into cancer in Norfolk (EPIC-Norfolk) Prospective Population Study. Circulation 2007;116:2694-701.

18. Shores MM, Matsumoto AM, Sloan KL, et al. Low serum testosterone and mortality in male veterans. Arch Intern Med 2006;166:1660-5.

19. Laughlin GA, Barrett-Connor E, Bergstrom J. Low serum testosterone and mortality in older men. J Clin Endocrinol Metab 2008;93:68-75.

20. English KM, Mandour 0, Steeds RP, et al. Men with coronary artery disease have lower levels of androgens than men with normal coronary angiograms. Eur Heart $J$ 2000;21:890-4.

21. Malkin CJ, Pugh PJ, West JN, et al. Testosterone therapy in men with moderate severity heart failure: a double-blind randomized placebo controlled trial. Eur Heart J 2006:27:57-64.

22. Diver MJ. Analytical and physiological factors affecting the interpretation of serum testosterone concentration in men. Ann Clin Biochem 2006;43:3-12

23. Wang C, Nieschlag E, Swerdloff R, et al. Investigation, treatment and monitoring of late-onset hypogonadism in males: ISA, ISSAM, EAU, EAA and ASA recommendations. Eur J Endocrinol 2008:159:507-14.

24. Tremblay RR, Dube JY. Plasma concentrations of free and non-TeBG bound testosterone in women on oral contraceptives. Contraception 1974;10:599-605.

25. Phillips GB, Pinkernell BH, Jing TY. The association of hypotestosteronemia with coronary artery disease in men. Arterioscler Thromb 1994;14:701-6.

26. van den Beld AW, Bots ML, Janssen JA, et al. Endogenous hormones and carotid atherosclerosis in elderly men. Am J Epidemiol 2003:157:25-31.

27. Hak AE, Witteman JC, de Jong FH, et al. Low levels of endogenous androgens increase the risk of atherosclerosis in elderly men: the Rotterdam study. $J$ Clin Endocrinol Metab 2002:87:3632-9.

28. Muller M, van den Beld AW, Bots ML, et al. Endogenous sex hormones and progression of carotid atherosclerosis in elderly men. Circulation 2004;109:2074-9.

29. Vermeulen A, Verdonck L, Kaufman JM. A critical evaluation of simple methods for the estimation of free testosterone in serum. J Clin Endocrinol Metab 1999:84:3666-72

30. Bhasin S, Singh $A B$, Mac RP, et al. Managing the risks of prostate disease during testosterone replacement therapy in older men: recommendations for a standardized monitoring plan. J Androl 2003;24:299-311. 